\title{
Pantoea Agglomerans Bacteremia: A Real Pathogen? A Case Report and Review of the Literature
}

\author{
Marco Bongiovanni* and Alessandro Maria Marra \\ ${ }^{\star}$ Corresponding author: Marco Bongiovanni, Pneumology Unit, Department of Medicine, Ospedale Salvini, Garbagnate Milanese, ASST Rhodense, Milan, Italy; \\ Mobile: +39 3335895304; Email: mbongiovanni@asst-rhodense.it
}

Received: May 04, 2021; Accepted: May 07, 2021; Published: May 13, 2021

\section{Introduction}

Pantoea agglomerans is a facultative anaerobic Gram-negative bacillus that can rarely cause opportunistic infections in humans, mainly due to wound infection with plant material or to hospitalacquired infections mostly in immune-compromised individuals. The clinical findings are extremely variable and reports of such an infection have been published mainly in premature infants or in oncologic individuals $[1,2]$. Its role as real human pathogen is still controversial due to infrequent reports of spontaneously occurring Pantoea agglomerans infections and uncertain in taxonomic identification $[3,4]$. We report here a case of Pantoea agglomerans bacteriemia in a subject with a Buerger's disease.

\section{Case presentation}

A 66 years-old man was admitted to our emergency department for fever up to $40^{\circ} \mathrm{C}$ after administration of intravenous prostaglandin analogs as therapy for Buerger's disease. He was carrier of a peripherally inserted catheter central (PICC) and affected by diabetes mellitus and chronic kidney failure. Serum level of CRP was 8,40 mg/ $\mathrm{dl}$ (normal value $<2 \mathrm{mg} / \mathrm{dL}$ ) and procalcitonine was $31 \mathrm{ng} / \mathrm{ml}$ (normal value $<0,5 \mathrm{ng} / \mathrm{ml})$. White blood cells were moderate augmented $(12000 / \mathrm{mmc}$, normal values $4000-11000 / \mathrm{mmc})$; renal and liver tests were normal, except for creatinine that was slightly increased $(1,4 \mathrm{mg} / \mathrm{dL}$, normale values $<1,2 \mathrm{mg} / \mathrm{dL})$. Chest X-ray was normal, as were two nasopharyngeal swabs for COVID-19 infection in two consecutive days. Blood cultures were promptly done and empiric antibiotic treatment with vancomycin was started due to the high risk of bacteremia due to meticillin-resistant Staphylococcus aureus. PICC was removed at the first day of hospitalization, supposing to be the possible source of infection. The patients had only a partial clinical and biochemical improvement after initial antibiotic treatment, with CRP $6,3 \mathrm{mg} / \mathrm{dL}$ at the second day of hospitalization. Abdomen ultrasound was normal and echocardiogram did not show findings compatible with endocarditis. On the third day after hospitalization, blood cultures resulted positive for Pantoea agglomerans; based on antibiogram, vancomycin was substituted with levofloxacin $750 \mathrm{mg}$. After further questioning, the patient revealed that he had recently done some garden works and had minor injuries to the legs that were not considered at the time of admission. Fever disappeared after 24 hours, and inflammatory parameters normalized after 72 hours. Patient was discharged after additional 5 days with a prescription of oral levofloxacin; levofloxacin was continued for a total of 14 days. After 2 months of follow-up no other febrile episodes recurred, and the patient maintains asymptomatic.

\section{Discussion}

Pantoea agglomerans, previously named Enterobacter agglomerans or Erwinia herbicola, is a non-capsulated, non-spore-forming anaerobic Gram-negative bacillus, usually associated with plants but rarely affecting humans and vertebrate animals. Approximately 20 species belong to this group of bacteria, and Pantoea agglomerans is the most prominent species in humans [5]. Clinical findings of patients infected by Pantoea agglomerans are very different, including bone and joints infections, osteomyelitis and synovitis [6]; uncommon presentation included endocarditis and endophtalmitis $[7,8]$. Usually, cutaneous infections occur as a wound super-infection, with the bacteria entering the skin when penetrating trauma occurs. From the skin, the infection can subsequently extend deep into the bones, causing septic arthritis, spondylodiscitis or osteomyelitis, theoretically leading to peritonitis or sepsis [9]. Other than wound infection with plant material, also exposure to contaminated fluids or medical equipment may lead to infection outbreaks $[10,11]$. However, sporadically Pantoea agglomerans can cause spontaneous bacteremia. The association between this finding and the gastroesophageal reflux disease or the receipt of antacids has been hypothesized, maybe due to gastrointestinal translocation following ingestion of bacteria with vegetables or fruits [12]. Finally, bloodstream infections by Pantoea agglomerans have been associated with occupational exposure to organic dust [13]. Overall, spontaneous bacteremia has been associated with several underlying pathologies like active malignancies, diabetes mellitus, chronic viral hepatitis, congestive heart failure, autoimmune or connettive diseases, cerebrovascular accidents, chronic pulmonary obstructive diseases and end-stage kidney diseases. The diagnosis of Pantoea agglomerans infection is usually made with positive cultures from different specimens including blood, pus, urine or tracheal aspirate. Regarding the effective antibiotic treatment, antimicrobial susceptibility was studied in a cohort of adult individuals developing a spontaneous bacteremia due to Pantoea agglomerans [12]. All the isolates were susceptible to ciprofloxacin, gentamicin, amikacin, piperacillin/tazobactam, cefotaxime, ceftazidime and imipenem; $61 \%$ were susceptible to cefazolin, $56 \%$ to ampicillin and $33 \%$ to fosfomycin. In our patient, levofloxacin treatment showed to be highly effective in a very short time, leading to a rapid recovery. As a consequence, the 
use of appropriate antibiotic treatment, driven by the susceptibility tests in vitro, also in immune-compromised individuals, is usually associated with a therapeutic success.

The possible cause of Pantoea agglomerans bacteremia in our patient was a wound superinfection; further he had several underlying clinical conditions such as a vasculitis like Buerger's disease, diabetes mellitus and chronic kidney failure that overall can have exacerbate the symptoms, contributing to the development of spontaneous bacteremia. However, the clinical course was well controlled by antibiotic treatment and no complications were observed in a medium-time.

\section{References}

1. Bergman KA, Arends JP, Scholvinck EH et al. (2007) Pantoea agglomerans septicemia in three newborn infants. Pediatr Infect Dis J 26(5):453-4. [cross-ref]

2. Yablon BR, Dantes R, Tsai V, et al. (2012-2013 \& 2017) Outbreak of Pantoea agglomerans bloodstream infections at an Oncology Clinic - Illinois. Infect Control Hosp Epidemiol 38(3):314-19. [cross-ref]

3. Rezzonico F, Smits TH, Montesinos E, et al.(2009) Genotypic comparison of Pantoea agglomerans plant and clinical strain. BMC Microbiol 9:204. [cross-ref]

4. Volksch B, Thon S, Jacobsen ID, et al. (2009) Polyphasic study of plant and clinicassociated Pantoea agglomerans strains reveals indistinguishable virulence potential. Infect Genet Evol 9:1381-91. [cross-ref]
5. Walterson AM, Stavrinides J. (2015) Pantoea: insights into a highly versatile and diverse genus within the enterobacteriaceae. FEMS Microbiology Reviews 39(6):968984. [cross-ref]

6. Flatauer FE. (1978) Septic arthritis caused by Enterobacter agglomerans. Arch Int Med 138(5):788. [cross-ref]

7. Williams AJK, Scott RJD, Lightfoot NF. (1986) Erwinia herbicola as a cause of bacterial endocarditis. J Infect 12(1):71-73. [cross-ref]

8. Mason GI, Bottone EJ, Podos SM. (1976) Traumatic endophtalmitis caused by an Erwinia species. Am J Ophtalmol 82(5):709-13. [cross-ref]

9. Okwundu N, Mercer J. (2019) Pantoea agglomerans cutaneous infection. J Derm and Dermatologic Surg 23(1):41.

10. Habsah H, Zeehaida M, Van Rostenberghe H, et al.(2005) An outbreak of Pantoea species in a neonatal intensive care unit secondary to contaminated parenteral nutrition. J Hosp Infect 61(3):213-18.

11. Rasmussen SW, Koczulab B. (1992) Transfusion associated bacteremia and septic shock due to Erwinia herbicola. Scand J Infect Dis 24(2):241-43. [cross-ref]

12. Cheng A, Liu CY, Tsai HY, et al. (2000-2010 \& 2013) Bacteremia caused by Pantoea agglomerans at a medical center in Taiwan, J Microbiol, Immunol and Infect 46(3):18794. [cross-ref]

13. Dutkiewicz J, Mackiewicz B, Lemieszek M, et al. (2015) Pantoea agglomerans: a marvelous bacterium of evil and good. Part I. Deleterious effects:dust-borne endotoxins and allergens-focus on cotton dust. Ann Agricol Environ Med 22(4):57688. [cross-ref]

\section{Citation:}

Marco Bongiovanni and Alessandro Maria Marra (2021) Pantoea Agglomerans Bacteremia: A Real Pathogen? A Case Report and Review of the Literature. Prev Med Epid Public Heal Volume 2(2): 1-2. 